PROCEEDINGS OF THE

AMERICAN MATHEMATICAL SOCIETY

Volume 49, Number 2, June 1975

\title{
SCHUR'S THEOREM FOR NEARLY KÄHLER MANIFOLDS
}

\author{
A. M. NAVEIRA AND L. M. HERVELLA
}

ABSTRACT. The classical theorem of Schur on Kähler manifolds is generalized to nearly Kähler manifolds, thus solving a conjecture of

A. Gray [3, p. 289].

Let $M$ be a $C^{\infty}$ manifold with metric tensor $g$, Riemannian connection $\nabla$, and almost complex structure $J$. Denote by $F(M)$ the real valued $C^{\infty}$ functions on $M$, by $\chi(M)$ the $C^{\infty}$ vector fields of $M$, and by $M_{m}$ the tangent space of $M$ at $m$. Then $M$ is said to be a nearly Kähler manifold provided $\left(\nabla_{X} J\right) X=0 \forall X \in \chi^{(M)}$. In this paper we consider $M$ as a nearly Kähler manifold.

Lemma 1 [2]. For all $W, X, Y, Z \in \chi(M)$, we have

$$
\begin{gathered}
R(X, Y, X, Y)-R(X, Y, J X, J Y)=\left\|\left(\nabla_{X} J\right) Y\right\|^{2}, \\
R(W, X, Y, Z)=R(J W, J X, J Y, J Z) .
\end{gathered}
$$

Lemma 2 [3]. For all $W, X, Y, Z \in \chi(M)$, we bave

$$
R(W, X, Y, Z)-R(W, X, J Y, J Z)=g\left\{\left(\nabla_{W} J\right) X,\left(\nabla_{Y} J\right) Z\right\} .
$$

Proposition 3. Let $R$ and $T$ be two quadrilinear mappings satisfying

$$
R(X, Y, Z, W)=-R(Y, X, Z, W)=-R(X, Y, W, Z)=R(Z, W, X, Y),
$$

$$
\begin{gathered}
R(X, Y, Z, W)+R(X, W, Y, Z)+R(X, Z, W, Y)=0, \\
R(X, Y, Z, W)-R(J X, J Y, Z, W)=g\left\{\left(\nabla_{X} J\right) Y,\left(\nabla_{Z} J\right) W\right\} .
\end{gathered}
$$

If $R(X, J X, X, J X)=T(X, J X, X, J X)$ for all $X \in M_{m}$, then

$$
\begin{aligned}
T(X, Y, Z, W)= & R(X, Y, Z, W) \\
& \pm\left[1 / 2 g\left\{\left(\nabla_{X} J\right) Y,\left(\nabla_{Z} J\right) W\right\}+1 / 4 g\left\{\left(\nabla_{Z} J\right) Y,\left(\nabla_{X} J\right) W\right\}\right. \\
& \left.-1 / 4 g\left\{\left(\nabla_{X} J\right) Z,\left(\nabla_{W} J\right) Y\right\}\right] .
\end{aligned}
$$

Received by the editors February 1, 1974 and, in revised form, March 20, 1974. AMS (MOS) subject classifications (1970). Primary 53C 55.

Key words and phrases. Nearly Kähler manifolds, Schur's theorem. 
Proof. We may assume that $T=0$. Consider $R-T$ and 0 instead of $R$ and $T$. Consider the quadrilinear mapping which sends $(X, Y, Z, W) \in M_{m}$ into

$$
\begin{aligned}
& R(X, J Y, Z, J W)+R(X, J Z, Y, J W)+R(X, J W, Y, J Z) \\
& \quad-1 / 2 g\left\{\left(\nabla_{X} J\right) Y,\left(\nabla_{Z} J\right) W\right\}-1 / 2 g\left\{\left(\nabla_{X} J\right) Z,\left(\nabla_{Y} J\right) W\right\}-1 / 2 g\left\{\left(\nabla_{X} J\right) W,\left(\nabla_{Y} J\right) Z\right\} .
\end{aligned}
$$

This quadrilinear mapping is symmetric in $X, Y, Z$ and $W$. Since it vanishes for $X=Y=Z=W$ by the assumption that $T=0$, it must vanish identically. Setting $X=Z$ and $Y=W$, we obtain

$$
2 R(X, J Y, X, J Y)+R(X, J X, Y, J Y)=g\left\{\left(\nabla_{X} J\right) Y,\left(\nabla_{X} J\right) Y\right\} .
$$

From (I), we have

$$
3 R(X, J Y, X, J Y)+R(X, Y, X, Y)=3 g\left\{\left(\nabla_{X} J\right) Y,\left(\nabla_{X} J\right) Y\right\} .
$$

Replacing $Y$ by $J Y$ in (II) and operating, we obtain

(III) $\quad-9 R(X, Y, X, Y)-3 R(X, J Y, X, J Y)=-9 g\left\{\left(\nabla_{X} J\right) Y,\left(\nabla_{X} J\right) Y\right\}$.

From (II) and (III), we obtain

$$
R(X, Y, X, Y)=3 / 4 g\left\{\left(\nabla_{X} J\right) Y,\left(\nabla_{X} J\right) Y\right\} .
$$

By a typical method we have

$$
\begin{aligned}
R(X, Y, Z, W)= & 1 / 2 g\left\{\left(\nabla_{X} J\right) Y,\left(\nabla_{Z} J\right) W\right\} \\
& +1 / 4 g\left\{\left(\nabla_{Z} J\right) Y,\left(\nabla_{X} J\right) W\right\}-1 / 4 g\left\{\left(\nabla_{X} J\right) Z,\left(\nabla_{W} J\right) Y\right\} .
\end{aligned}
$$

Now we define the quadrilinear mapping $R_{0}^{\prime}$ as follows:

$$
\begin{aligned}
R_{0}^{\prime}(X, Y, Z, W)= & g(X, Z) g(Y, W)-g(X, W) g(Y, Z)+g(X, J Z) g(Y, J W) \\
& -g(X, J W) g(Y, J Z)+2 g(X, J Y) g(Z, J W) \\
& +1 / 2 g\left\{\left(\nabla_{X} J\right) Y,\left(\nabla_{W} J\right) Z\right\}-1 / 4 g\left\{\left(\nabla_{Z} J\right) Y,\left(\nabla_{X} J\right) W\right\} \\
& +1 / 4 g\left\{\left(\nabla_{X} J\right) Z,\left(\nabla_{W} J\right) Y\right\} .
\end{aligned}
$$

Proposition 4. The quadrilinear mapping $R_{0}^{\prime}$ satisfies (I), and

$$
R_{0}^{\prime}(X, J X, X, J X)=\|X\|^{2} .
$$

Proposition 5. Let $P$ be a plane at $M$; let $X, Y$ be an orthonormal 
basis for $P ; K(P)$ their sectional curvature; $R$ a quadrilinear mapping satisfying (I). If $K(P)$ is constant for all planes $P$, invariant by $J$, then

$$
\begin{aligned}
R(X, Y, Z, W)= & K R_{0}^{\prime}(X, Y, Z, W) \\
& \pm\left[1 / 2 g\left\{\left(\nabla_{X} J\right) Y,\left(\nabla_{Z} J\right) W\right\}+1 / 4 g\left\{\left(\nabla_{Z} J\right) Y,\left(\nabla_{X} J\right) W\right\}\right. \\
& \left.\quad-1 / 4 g\left\{\left(\nabla_{X} J\right) Z,\left(\nabla_{W} J\right) Y\right\}\right] .
\end{aligned}
$$

The proof is immediate from Lemma 2 and Proposition 3.

Theorem 6. Let $M$ be a connected nearly Käbler manifold of complex dimension $n \geq 2$. If the bolomorphic sectional curvature $K(P)$, where $P$ is a plane in $M_{m}$ invariant by $J$, depends only on $m$, then $M$ is a space of constant bolomorphic sectional curvature.

Proof. We denote by $a(X, Y, Z, W)$ the tensor

$$
\begin{aligned}
\alpha(X, Y, Z, W)= & 1 / 2 g\left\{\left(\nabla_{X} J\right) Y,\left(\nabla_{Z} J\right) W\right\} \\
& +1 / 4 g\left\{\left(\nabla_{Z} J\right) Y,\left(\nabla_{X} J\right) W\right\}-1 / 4 g\left\{\left(\nabla_{X} J\right) Z,\left(\nabla_{W} J\right) Y\right\} .
\end{aligned}
$$

By. Proposition 5, we know that

$$
\begin{aligned}
R(X, Y, Z, W) & =K R_{0}^{\prime}(X, Y, Z, W) \pm \alpha(X, Y, Z, W) \\
& =K R_{0}(X, Y, Z, W)-(K \pm 1) \alpha(X, Y, Z, W),
\end{aligned}
$$

where

$$
\begin{aligned}
R_{0}(X, Y, Z, W)= & g(X, Z) g(Y, W)-g(X, W) g(Y, Z)+g(X, J Z) g(Y, J W) \\
& -g(X, J W) g(Y, J Z)+2 g(X, J Y) g(Z, J W) .
\end{aligned}
$$

From (VI), we obtain

$\left(\nabla_{U} R\right)(X, Y, Z, W)=(U K) R_{0}(X, Y, Z, W)+K\left(\nabla_{U} R_{0}\right)(X, Y, Z, W)$

$$
-(U K) \alpha(X, Y, Z, W)-(K \pm 1)\left(\nabla_{U} \alpha\right)(X, Y, Z, W) \text {. }
$$

If we make the cyclic sum in (VII) with respect to $(U, X, Y)$ and use Bianchi's second identity, we obtain

$$
\begin{aligned}
0= & (U K)\left(R_{0}-\alpha\right)(X, Y, Z, W) \\
& +(Y K)\left(R_{0}-\alpha\right)(U, X, Z, W)+(X K)\left(R_{0}-\alpha\right)(Y, U, Z, W) \\
& +K\left\{\left(\nabla_{U} R_{0}\right)(X, Y, Z, W)+\left(\nabla_{Y} R_{0}\right)(U, X, Z, W)+\left(\nabla_{X} R_{0}\right)(Y, U, Z, W)\right\} \\
& -(K \pm 1)\left\{\left(\nabla_{U} \alpha\right)(X, Y, Z, W)+\left(\nabla_{Y} \alpha\right)(U, X, Z, W)+\left(\nabla_{X} \alpha\right)(Y, U, Z, W)\right\} .
\end{aligned}
$$


For an arbitrary $Y$, we choose $X$ such that $X, Y, J Y$ are mutually orthogena! to $Y, J Y$ and $X$. Besides we can choose $U, Z$ and $W^{\prime}$ such that $U=Z=$ $J X, W=X$. Then

$$
\begin{aligned}
0= & 4(Y K) g(X, X)^{2} \\
& +K\left\{\left(\nabla_{J X} R_{0}\right)(X, Y, J X, X)+\left(\nabla_{Y} R_{0}\right)(J X, X, J X, X)\right. \\
& \left.+\left(\nabla_{X} R_{0}\right)(Y, J X, J X, X)\right\} \\
& -(K \pm 1)\left\{\left(\nabla_{J X} \alpha\right)(X, Y, J X, X)+\left(\nabla_{Y} \alpha\right)(J X, X, J X, X)\right. \\
& \left.+\left(\nabla_{X} \alpha\right)(Y, J X, J X, X)\right\} .
\end{aligned}
$$

By using $\left(\nabla_{X} J\right) X=0$ and $g\left(\left(\nabla_{Y} J\right) X, X\right)=0$ for $Y$ arbitrary, from

$$
\begin{aligned}
\left(\nabla_{U} R_{0}\right)(X, Y, Z, W)= & \nabla_{U} R_{0}(X, Y, Z, W)-R_{0}\left(\nabla_{U} X, Y, Z, W\right) \\
& -R_{0}\left(X, \nabla_{U} Y, Z, W\right)-R_{0}\left(X, Y, \nabla_{U} Z, W\right) \\
& -R_{0}\left(X, Y, Z, \nabla_{U} W\right)
\end{aligned}
$$

and

$$
\begin{aligned}
\left(\nabla_{U} \alpha\right)(X, Y ; Z, W)= & \nabla_{U} \alpha(X, Y, Z, W)-\alpha\left(\nabla_{U} X, Y, Z, W\right) \\
& -\alpha\left(X, \nabla_{U} Y, Z, W\right)-\alpha\left(X, Y, \nabla_{U} Z, W\right) \\
& -\alpha\left(X, Y, Z, \nabla_{U} W\right),
\end{aligned}
$$

by substituting and operating, we prove that

$$
\begin{aligned}
\left(\nabla_{J X} R_{0}\right)(X, Y, J X, X) & =\left(\nabla_{Y} R_{0}\right)(J X, X, J X, X) \\
& =\left(\nabla_{X} R_{0}\right)(Y, J X, J X, X)=0
\end{aligned}
$$

and

$$
\left(\nabla_{J X^{\alpha}} \alpha\right)(X, Y, J X, X)=\left(\nabla_{Y}^{\alpha}\right)(J X, X, J X, X)=\left(\nabla_{X^{\alpha}}\right)(Y, J X, J X, X)=0 .
$$

Then $(Y K)=0$.

\section{BIBLIOGR APHY}

1. A. Gray, Minimal varieties and almost Hermitian submanifolds, Michigan Math. J. 12 (1965), 273-287. MR 32\#1658.

2. - Almost complex submanifolds of the six sphere, Proc. Amer. Math. Soc. 20 (1969), 277-279. MR 39 \#7636.

3. - Nearly Kähler manifolds, J. Differential Geometry 4 (1970), 283309. MR $42 \# 2404$. 
4. S. Kobayashi and K. Nomizu, Foundations of differential geometry. Vol. I, Interscience Tracts in Pure and Appl. Math., no. 15, Interscience, New York, 1963; Vol. II, 1969. MR 27 \#2945; 38 \#6501.

5. A. M. Naveira, Caracterisation des variétés à courbures sectionnelles holomorphes généralizées constantes, J. Differential Geometry 9 (1974), 55-60.

DEPART AMENTO DE GEOMETRIA Y TOPOLOGIA, UNIVERSIDAD DE SANTIAGO, SANTIAGO DE DOMPOSTELA, SPAIN 